\title{
CRITERIOS DE EVALUACIÓN DE LA CALIDAD DE LAS ARGUMENTACIONES POR ESTUDIANTES UNIVERSITARIOS BRASILEROS Y COLOMBIANOS ${ }^{1}$
}

\author{
ASSESSMENT CRITERIA OF THE QUALITY \\ OF THE ARGUMENTS BY BRAZILIAN AND \\ COLOMBIAN UNIVERSITY STUDENTS
}

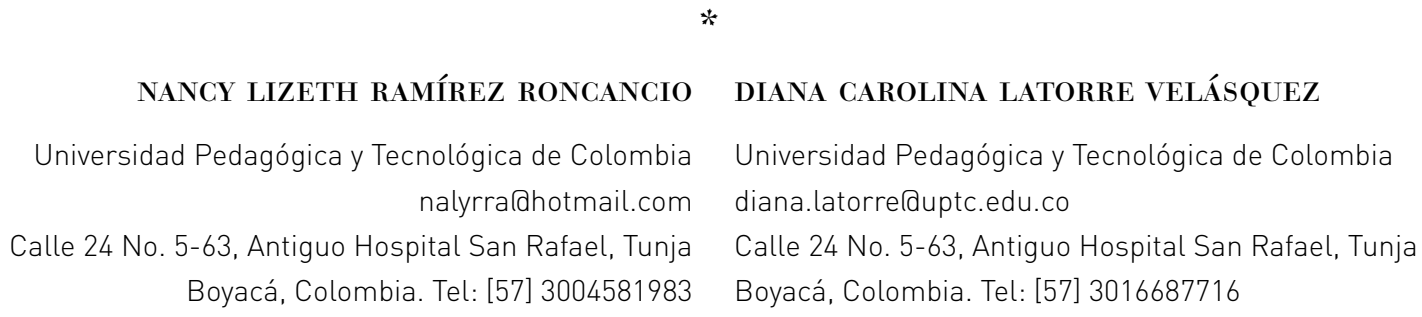

El presente artículo problematiza sobre la evaluación de la argumentación como proceso complejo que involucra el examen de aspectos estructurales y validez del argumento, así como la situación comunicativa y las características del argumentador. Tiene como objetivo analizar el uso de criterios de evaluación de la calidad de las argumentaciones en estudiantes universitarios de Brasil y Colombia. Utilizó una metodología cualitativa para el análisis de una serie de casos, cinco estudiantes universitarios de psicología brasileños y cinco colombianos, escogidos a través de muestreo intencional. La construcción de datos se realizó en contexto de aula orientado al desarrollo de competencias argumentativas (identificación, producción y evaluación) y la construcción de conocimiento curricular. El corpus de análisis correspondió a evaluaciones online realizadas por estudiantes universitarios mientras examinan las argumentaciones producidas por sus colegas durante situaciones de debate crítico. Los hallazgos muestran comunalidades y

\footnotetext{
1 Estudio realizado usando los datos y las categorías analíticas de la disertación de maestría de la primera autora y de la tesis de doctorado de la segunda autora. Trabajos realizados bajo la asesoría de la PhD. Selma Leitão, con financiamiento del Consejo Nacional de Pesquisa - CNPq y de la PEC-PG - Brasil.
} 
diferencias, en cuanto al empleo de criterios de calidad del argumento (dialécticos, estructurales) $y$ de la argumentación (pragmáticos y reflexivos) en los casos analizados de los dos países. Se evidencian transformaciones intra e interindividuales en la utilización de criterios para emitir juicios sobre los argumentos y las argumentaciones producidas por otras personas.

Palabras Clave: evaluación, criterios de evaluación, argumentación, contexto universitario.

The evaluation of intellectual argumentation processes is analyzed as a complex endeavor involving the validity and structure of arguments, the communication setting and the personal characteristics of the argumentator. Thus, the objective was set to assess the criteria employed by Brazilian and Colombian university students to evaluate the argumentation quality of their classmates. A qualitative methodology was applied to the study of five Brazilian and five Colombian psychology students, selected by intentional sampling. The corpus data were obtained in a classroom environment designed to develop argumentative competencies (identification, production, and evaluation) and academic knowledge. The focus of analysis relates to online evaluations carried out during situations of critical debate. The findings show similarities and differences across the studied countries and individuals regarding the criteria employed to evaluate argument (dialectical, structural) and argumentation (pragmatic and reflexive) quality. Intra and inter-personal transformations serve as evidence in the use of these criteria.

Key words: evaluation, evaluation criteria, argumentation, university context. 


\section{Introducción}

La teoría de argumentación establece distinciones entre como comprender la argumentación y el argumento. Las diferencias radican fundamentalmente, en que la argumentación, involucra a la persona argumentadora y la práctica de argumentar, por tanto, es considerada como un proceso de intercambio dialógico a través del cual un argumentador se compromete con la defensa de un punto de vista y se contrapone a un otro, real o ideal, con el fin de poner a prueba la plausibilidad de sus posicionamientos. Mientras que, el argumento es definido como un producto del proceso y de la práctica argumentativa, el cual responde a una estructura lógica que tiene como fin ofrecer razones para una conclusión (Govier, 1987; Halpern, 1998; 2003; Johnson, 2000; Marraud, 2007; Rapanta, García-Mila y Gilabert, 2013; Saíz, 2002; Toulmin, 1980).

La anterior distinción, junto con el reconocimiento al papel que juega la argumentación al activar el mecanismo de revisión de perspectivas (Kuhn y Udell, 2003; Leitão, 2007; Saíz, 2002), el proceso dialógico de ideas opuestas, y las características del argumentador, conllevan a comprender la evaluación como un proceso metacognitivo. Proceso a través del cual, se ofrecen juicios producto de una acción deliberativa (Govier, 2014; Lipman, 2003) que convoca al sujeto a realizar la reflexión sobre sus posiciones y sobre las posiciones de otros. Es decir, lo lleva a que adopte como foco de análisis sus propias concepciones sobre el mundo (Leitão, 2007) y al hacerlo, este instaura criterios que orientan la validez de su juzgamiento (Halpern, 2014; Kuhn, 1991; 1999; Lipman, 2003; Rapanta et al., 2013).

El proceso evaluativo involucra el examen objetivo y sistemático a través de criterios o parámetros que son usados para construir juicios y guiar el acto deliberativo (Lipman, 2003). En ese sentido, el uso de criterios en la evaluación de la argumentación es un tema de interés en diferentes ámbitos como, por ejemplo, la teoría de la argumentación y la psicología, desde los cuales se ha propuesto criterios orientados a evaluar; la estructura del argumento, la validez de las justificaciones y la relación que existe entre el punto de vista y las razones que lo sustentan (Gaviria y Corredor, 2011; Govier, 2014; Kuhn, 1991; Kuhn y Udell, 2003; Van Eemeren, Grootendorst y Snoeck Henkemans, 2008).

La evaluación por criterios considera no solo la calidad del argumento, o de la información usada como apoyo para un punto de vista, sino también, el contexto en que esta ocurre, la práctica de argumentar y al argumentador. De modo que, cuando se evalúa, se debe considerar, el grado de cogencia del raciocinio (Govier, 2010; Halpern, 2014; Saíz y Rivas, 2008), las circunstancias particulares en que la argumentación ocurre (persona y contexto), las características de comunicación y 
discusión crítica (Van Eemeren, Grootendorst, 2004), y la reflexividad del pensamiento (Latorre, 2018). Aspectos, a través de las cuales, es posible comprender las transformaciones de un sujeto en su competencia para evaluar la argumentación de otros (Latorre, 2018; Ramírez, 2012; Ramírez, Souza y Leitão, 2013). Al referirnos a la cogencia del raciocinio, se adopta, por un lado, los indicadores para evaluar la estructura del argumento desde la perspectiva de Kuhn (2003) y, por otro lado, los criterios de calidad del argumento - aceptabilidad, relevancia y suficiencia - propuestos por Govier (2010). El criterio de aceptabilidad tiene como fin la evaluación sobre la plausibilidad (verdad) de las justificaciones usadas en apoyo al punto de vista; el de relevancia examina la relación lógica entre las razones ofrecidas y el punto de vista; el de suficiencia valora sí las justificaciones usadas, en conjunto, proporcionan un apoyo racional al punto de vista. Asimismo, al aludir las características de comunicación y discusión crítica, se toma como referencia los criterios pragmáticos propuestos por Van Eemeren et al. (2008), que enfatizan la práctica razonable de la argumentación, sin desconocer la presencia de acciones dirigidas a convencer a la audiencia cuando se ofrecen argumentos. Finalmente, al mencionar la reflexividad del pensamiento, se adopta, en primer lugar, las comprensiones de Leitão (2007), quien resalta las características reflexivas de la argumentación cuando un sujeto toma sus propios puntos de vista como objeto de pensamiento $y$, considera las bases en que estas se apoyan para generar un cambio, o no, sobre sus concepciones. En segundo lugar, a lo planteado por Carraher (1983) acerca de tener apertura para considerar diferentes puntos de vista y no parcializarse o sesgarse por una perspectiva.

\section{¿Qué sabemos acerca de la utilización de criterios para evaluar argumentos?}

A pesar de existir interés en evaluar el argumento, aun son pocas las investigaciones preocupadas por estudiar el uso de criterios en el proceso argumentativo. La evaluación de argumentos y argumentaciones es un proceso complejo que no solo refleja elementos normativos del argumento, sino que, también, involucra las condiciones propias del contexto en que la argumentación es producida y las características de los argumentadores. Aspectos que están permeados por los conocimientos previos, las creencias, los valores culturalmente construidos, los cuales pueden flexibilizar o no, tanto el examen que se hace sobre los propios puntos de vista, como de los puntos de vista ofrecidos por los otros (Walton, 1992; 2010).

Las investigaciones privilegian la evaluación de situaciones argumentativas de producción de argumentos, orientadas a examinar la calidad de la estructura del argumento, la validez de las razones en sí y la relevancia entre el punto de vista y la justificación. Al examinar la calidad de la estructura de los argumentos produci- 
dos por estudiantes, los investigadores notan la presencia de justificaciones simples debido a la dificultad para coordinar las evidencias en sus justificaciones; además, observan que la motivación y la atención se convierten en factores que afectan el uso de las evidencias y, en consecuencia, la construcción de argumentos más sofisticados (Clark y Sampson, 2008).

Los estudios que discuten la validez de los argumentos basados en la evaluación de sus evidencias permiten verificar las dificultades que existen para distinguir la naturaleza epistémica de las justificaciones, lo que conlleva a presentar dificultades en el entendimiento explícito de la validez de la evidencia y la fuerza del argumento (Felton y Kuhn, 2001; Kuhn y Pearsall, 2000). Sin embargo, Barchfeld y Sodian (2009) sostienen que estas dificultades mejoran tanto con el avance en los años escolares como a partir de la participación en procesos de formación en argumentación (Gaviria y Corredor, 2011).

Los resultados de las investigaciones orientadas a evaluar la relevancia de los argumentos concluyen que los estudiantes presentan dificultades para evaluar las conexiones entre afirmaciones y razones (Larson, Britt y Kurby, 2009), asimismo, a pesar de participar en procesos de intervención para mejorar la producción de argumentos los estudiantes presentan problemas para reconocer la fuerza en los contraargumentos usados para debilitar el punto de vista opuesto (Goldstein, Crowell y Kuhn, 2009).

En conjunto, los hallazgos de los estudios citados muestran la complejidad y dificultades presentes en la evaluación de los argumentos y señalan el vacío existente en la literatura sobre el uso de criterios que abarquen la práctica argumentativa y las características del argumentador a la hora de examinar las argumentaciones de otras personas. Laguna en la cual este artículo pretende contribuir al consolidar los indicadores de evaluación que emergen en situaciones de argumentación natural de construcción de conocimiento, por ejemplo, el debate critico-reflexivo en torno a temas curriculares.

También, es evidente el uso de diseños experimentales en los que se evalúa argumentos producidos fuera del aula de clase (Britt, Kurby, Dandotkar y Wolfe, 2008; Glassner, Weinstock y Neuman, 2005; Felton y Kuhn, 2001; Larson et al., 2009; Wolfe, 2012) o con tópicos distantes del contenido curricular, como temas de naturaleza socio-científica, por ejemplo, el desempleo, la reincidencia en el crimen, entre otros (Kuhn, 2000; Kuhn y Pearsall, 2000). Contrario a esta línea, este estudio privilegia la práctica argumentativa en contextos educativos auténticos, facilitando la comprensión de la complejidad de la competencia evaluativa, en la que no solo 
adquiere relevancia los aspectos dialécticos propios del argumento sino también, aspectos de tipo pragmático y reflexivo.

Finalmente, la literatura muestra los inconvenientes existentes en el ámbito de la praxis educativa, específicamente, al articular las políticas educativas con la implementación de metodologías y estrategias pedagógicas orientadas a la enseñanza de competencias argumentativas al interior del aula (Cassiani, 2010; Lozano, 2010). Hecho que se corrobora, a nivel educativo, con las debilidades encontradas, por un lado, en estudiantes de secundaria y universitarios con respecto a habilidades cognitivas de análisis, evaluación, argumentación e interpretación (Causado-Escobar, Santos-Carrasco y Calderón-Salas, 2015; Programme for International Student Assessment [PISA], 2018; Instituto Colombiano para la Evaluación de la Educación [ICFES], 2017; Instituto Nacional de Estudos e Pesquisa Educacionais Anisio Texeira [Inep], 2019) y, por otro lado, en el ámbito de la praxis educativa, en el que se notan dificultades a la hora de implementar estrategias adecuadas dentro del aula que permitan favorecer el desarrollo de estas habilidades (Andrews, 2010; JiménezAlexandre y Erduran, 2007). Por tanto, aún persisten en el ámbito teórico y práctico cuestiones relacionadas sobre cómo desarrollar la competencia argumentativa orientadas tanto a la producción como la evaluación de argumentos. En este sentido, analizar el uso de criterios de evaluación en dos contextos diferentes permite identificar las fortalezas propias del design pedagógico, sobre la cual, pueda proponerse mejoras metodológicas que permitan mitigar las deficiencias encontradas en la praxis sobre el desarrollo de las competencias argumentativas.

En consecuencia, el objetivo de este estudio fue analizar el uso de criterios de evaluación de la calidad de las argumentaciones en estudiantes universitarios de Brasil y Colombia, involucrados en un escenario argumentativo. De modo que, a través del análisis se examine si existen diferencias y comunalidades en la utilización de los indicadores analíticos en el conjunto de casos elegido de los dos países. Asimismo, analizar cuáles son las transformaciones en el uso de criterios empleados a través de distintos momentos evaluativos.

\section{Método}

Investigación ideográfica de estudio de caso (Yin, 2002), con énfasis especial en el análisis de las producciones discursivas de estudiantes en el contexto da aula de clase. Su interés se centra en analizar en detalle múltiples casos, para comprender las posibles transformaciones ocurridas durante un periodo de tiempo (intervención pedagógica basada en argumentación), con respecto al uso de criterios de evaluación del argumento y de la argumentación en estudiantes universitarios. 


\section{Participantes}

Para el estudio se tomaron de forma intencional cinco casos de estudiantes brasileños y cinco colombianos que cursaban primer y tercer semestre de psicología, respectivamente, pertenecientes a universidades públicas de los dos países. La media de edad de los participantes brasileros fue de 20 años y la de los colombianos de 18 años. Los casos analizados fueron cuatro mujeres y un hombre, respectivamente de cada país.

Estos estudiantes participaron de una asignatura en la cual se implementó la estrategia pedagógica propuesta por Leitão (2012), cuyos objetivos son tanto la construcción de conocimiento como el desarrollo de pensamiento reflexivo sustentado en el uso de competencias argumentativas. Es una asignatura obligatoria en el currículo brasileño y electiva en el currículo colombiano, ambas son de naturaleza teórica. La intensidad horaria de las clases fue de 48 horas semanales en el contexto de aula colombiano y de 64 horas semanales en el contexto de aula brasileño. Como criterio de inclusión, se estableció que los participantes tuvieran mínimo tres evaluaciones online de debates críticos realizados en la asignatura, con el objetivo de analizar posibles transformaciones en cada uno de ellos. De igual manera, se tuvo en cuenta que dieran su consentimiento para participar de forma voluntaria en la investigación.

La recolección de los datos estuvo sujeta a las condiciones éticas establecidas en las leyes brasileñas (resolución CNS 196/1996) y colombianas (Ley del psicólogo 1090 de 2006 y (resolución No 8430 de 1993 del Ministerio de salud), que reglamenta el ejercicio profesional del psicólogo y las normas científicas, técnicas y administrativas de la investigación. De esta manera, se respetó el consentimiento informado de los participantes, garantizando la total confidencialidad y privacidad de su identidad, utilizando nombres ficticios. Los datos son usados exclusivamente para fines académicos y de investigación.

\section{Procedimiento}

Contexto de producción de los datos

La estrategia pedagógica propuesta por Leitão (2012) promueve el ejercicio colaborativo entre pares, estudiante-docente, estudiante-monitor para el desarrollo de competencias de identificación, producción y evaluación de argumentos. Asimismo, a través de la construcción de un escenario dialógico-discursivo que favorece el ejercicio de revisión de perspectivas propias y de otros, la ponderación de evidencias, la vigilancia epistémica, entre otros aspectos que propician el desarrollo cognitivo (Baker, 1996; Halpern, 2014; Kuhn, 1991; Leitão, 2000; 2008; 2012; Voss y Van Dyke, 2001) y metacognitivo (Brown, 1997; Crowell y Kuhn, 2014; Hemberger y Kuhn, 2018; Kuhn, 
Hemberger y Khait, 2016; Kuhn, Matos y Shi, 2017; Larraín, Freire, Moretti, Requena y Sabat, 2015; Meneses, Osorio y Rubio, 2017; Torres, Fonseca y Pineda, 2017).

Metodológicamente, la estrategia se organiza a través de ciclos constituidos por cuatro fases: familiarización, preparación, debate crítico, feedback y cierre, que se realizan sistemáticamente para abordar controversias propuestas en las asignaturas.

- La fase de familiarización presenta el contenido curricular al que se asocia la controversia; además, ofrece diferentes perspectivas de explicación y comprensión del tema tratado.

- La fase de preparación entrena sistemática y progresivamente en competencias tanto de producción como evaluación de argumentos que serán usados durante la fase de debate crítico. En relación con la construcción del conocimiento, esta fase refuerza la apropiación que los estudiantes tienen sobre el tema discutido. Pedagógicamente, se trabaja a través de grupos de discusión colaborativa para la construcción de la línea de raciocinio a seguir en el debate crítico, para ello, se realizan ejercicios de identificación y construcción de argumentos y contraargumentos, elaboración y consenso de criterios para evaluar el debate.

- La fase de debate crítico corresponde a un espacio de deliberación entre equipos - proponente, oponente e investigadores/jueces -, quienes evidencian los conocimientos construidos y las competencias argumentativas adquiridas hasta este momento. Esta fase se desarrolla a través de cinco etapas de debate crítico, que ocurren según lo planteado por Fuentes (2011) - contextualización, debate cerrado, debate abierto, fase de preguntas, conclusión y cierre evaluativo. Durante esta fase, los estudiantes deben realizar una evaluación online de lo ocurrido en cada una de las etapas del debate y dar su veredicto justificado (momento privilegiado para el recorte analítico).

- En la fase de feedback y cierre del ciclo, se realiza un proceso de retroalimentación del desempeño de los estudiantes durante el ciclo con respecto a dos aspectos, por un lado, la calidad del contenido construido y, por otro lado, el desarrollo de competencias argumentativas y de pensamiento reflexivo.

En cada ciclo los estudiantes asumen papeles diferentes con el fin de promover la revisión de perspectivas y ejercer roles de oposición, acciones que pueden garantizar mayor vigilancia epistémica, disminución en sesgos y misconceptions.

Los momentos seleccionados, para el análisis corresponden a los textos de evaluación online del debate crítico producidos en las asignaturas, en las que progresivamente, también, se incorporan y entrenan aspectos de la argumentación. 


\section{Análisis}

Corpus de Análisis

Como corpus de análisis fue utilizado el texto escrito de la evaluación online que realizaron los estudiantes de situaciones de debate crítico. Material usado para realizar el análisis discursivo y de identificación de criterios evaluativos. El total de evaluaciones de debate fue de 17 para Brasil y 19 para Colombia. Cada participante produjo entre 3 y 4 evaluaciones. No se encuentra uniformidad en el número de debates evaluados por los participantes, ya que esto dependió del diseño de la asignatura (no obligatoriedad de las actividades) y las características propias de la misma en cada país. Los temas de debate fueron diferentes en cada país, sin embargo, tuvieron en común el hecho de circunscribirse al ámbito de conocimiento psicológico y que son controversias genuinas en este campo del saber, caracterizadas por ser impases persistentes en el tiempo, poseer interpretaciones divergentes (diversas perspectivas), y su resolución (temporal) implica la ponderación de posiciones a través de la argumentación crítica (Van Eemeren y Garssen, 2008). La información introducida sobre argumentación fue la misma en los dos países. En la tabla 1, se presentan los temas abordados a nivel curricular y de argumentación.

\section{Tabla 1.}

Temas de debate crítico y de teoría de argumentación usados en Brasil y Colombia

\begin{tabular}{|c|c|c|}
\hline Temas & & Contenido argumentación \\
\hline Colombia & Brasil & \multirow{6}{*}{$\begin{array}{l}\text { Opinión vs Opinión fundamentada. } \\
\text { Estructura del argumento. } \\
\text { Tipos de Información y marcadores } \\
\text { textuales. } \\
\text { Criterios de evaluación }\end{array}$} \\
\hline $\begin{array}{l}\text { Soportes epistemológicos } \\
\text { Modelo Comportamental vs. } \\
\text { Modelo Cognitivo. }\end{array}$ & $\begin{array}{l}\text { Innato y adquirido en la formación } \\
\text { de la identidad de género. }\end{array}$ & \\
\hline $\begin{array}{l}\text { Principios Skinnerianos para } \\
\text { explicar la conduta verbal vs. } \\
\text { cognitivos y sociocognitivos. }\end{array}$ & $\begin{array}{l}\text { Estudio de la personalidad a través } \\
\text { de la teoría de rasgos }\end{array}$ & \\
\hline $\begin{array}{l}\text { La inteligencia es una habili- } \\
\text { dad general o específica }\end{array}$ & $\begin{array}{l}\text { Diferencias entre hombres y muje- } \\
\text { res alrededor de la inteligencia }\end{array}$ & \\
\hline \multirow{2}{*}{ Toma de decisiones } & $\begin{array}{l}\text { Relevancia de la Inteligencia Emo- } \\
\text { cional para el estudio del compor- } \\
\text { tamiento humano. }\end{array}$ & \\
\hline & $\begin{array}{l}\text { Perspectivas interaccionistas para } \\
\text { explicar la constitución de las dife- } \\
\text { rencias individuales. }\end{array}$ & \\
\hline
\end{tabular}

Nota. Fuente propia. 


\section{Procedimiento Analítico}

Para identificar el uso que estudiantes universitarios, de Brasil y Colombia, realizan de criterios de calidad de la argumentación y poder establecer las comunalidades y diferencias, fueron seguidos tres momentos: preanálisis, microanálisis y macroanálisis. El procedimiento analítico se realizó a través de revisión conjunta (investigadoras) y por un juez experto en argumentación.

El preanálisis, buscó identificar la presencia/ausencia de enunciados que indicaran movimientos evaluativos en los textos de los participantes. Para esto, en primer lugar, se preservó el formato de escritura de las evaluaciones realizadas por los participantes y, la secuencia de realización de las actividades, ya que estas no tuvieron un carácter obligatorio. En segundo lugar, se rastrearon pistas lingüísticas, tales como, modalizadores (bueno, malo), la semántica del término, el sentido del uso dado al criterio evaluativo de acuerdo con el contexto de producción.

El microanálisis, permitió capturar en detalle la presencia de indicadores de los criterios de evaluación utilizados por los participantes. Para esta identificación, se utilizaron los indicadores evaluativos propuestos por Latorre (2018); Ramírez et al. (2013) y Ramírez (2012), en investigaciones previas, quiénes proponen categorías analíticas diferenciando criterios usados para evaluar el argumento y la argumentación. Para el argumento, las autoras proponen categorías de naturaleza dialéctica (aceptabilidad, relevancia y suficiencia) y estructural (cantidad de justificaciones, elementos del argumento). Para la argumentación, plantean categorías de naturaleza reflexiva (pluralismo y flexibilidad del pensamiento) y pragmática (discursivos, retóricos, condiciones de producción). La Tabla 2, ofrece una descripción detallada de los criterios que orientaron el análisis. 


\section{Tabla 2}

Tipo de criterios usados en el análisis de la evaluación de la calidad de los argumentos y las argumentaciones

\begin{tabular}{|c|c|c|c|c|}
\hline \multicolumn{2}{|c|}{ Naturaleza } & Definición & Criterio & Indicadores de Uso \\
\hline \multirow{19}{*}{ 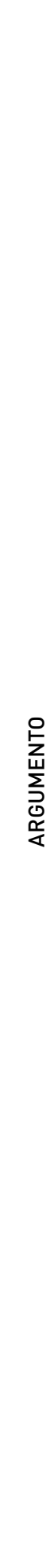 } & \multirow{17}{*}{ 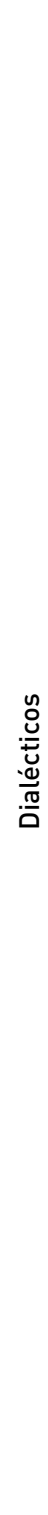 } & \multirow{17}{*}{$\begin{array}{l}\text { Evaluación de la } \\
\text { fuerza y la so- } \\
\text { lidez del argu- } \\
\text { mento (Govier, } \\
2014 \text { ). } \\
\end{array}$} & \multirow{15}{*}{ Aceptabilidad } & Evalúa la presencia o no de referencias. \\
\hline & & & & Evalúa la forma en que las referencias son citadas. \\
\hline & & & & Evalúa la presencia o no de estudios o experimentos. \\
\hline & & & & $\begin{array}{l}\text { Evalúa la confiabilidad de la evidencia científica (e.g., ins- } \\
\text { trumentos empleados, o procedimiento seguido). }\end{array}$ \\
\hline & & & & Evalúa la autoridad de la fuente. \\
\hline & & & & Evalúa el uso de datos o estadísticas. \\
\hline & & & & Evalúa la presencia de conocimiento de sentido común. \\
\hline & & & & $\begin{array}{l}\text { Evalúa la presencia de conocimiento previo o por expe- } \\
\text { riencia. }\end{array}$ \\
\hline & & & & Evalúa el uso de ejemplos. \\
\hline & & & & $\begin{array}{l}\text { Evalúa la cantidad (número) de fuentes o referencias } \\
\text { usadas. }\end{array}$ \\
\hline & & & & Evalúa el uso de conceptos técnicos. \\
\hline & & & & Evalúa el uso de información hipotética. \\
\hline & & & & Evalúa el uso de referencias alternas de información. \\
\hline & & & & Evalúa la plausibilidad de las informaciones. \\
\hline & & & & Crítica a una analogía. \\
\hline & & & Relevancia & $\begin{array}{l}\text { Evalúa la relación entre la(s) justificación(es) y el punto } \\
\text { de vista. }\end{array}$ \\
\hline & & & Suficiencia & $\begin{array}{l}\text { Evalúa la necesidad de presentar más justificaciones para } \\
\text { dar mayor soporte al punto de vista. }\end{array}$ \\
\hline & & & $\begin{array}{l}\text { Elementos del } \\
\text { Argumento }\end{array}$ & $\begin{array}{l}\text { Evalúa la presencia de un punto de vista y justificaciones } \\
\text { que lo sustentan como mínima estructura del argumento. }\end{array}$ \\
\hline & 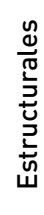 & $\begin{array}{l}\text { Evalúan la pre- } \\
\text { sencia de los } \\
\text { componentes } \\
\text { estructurantes } \\
\text { de un argu- } \\
\text { mento. }\end{array}$ & $\begin{array}{l}\text { Cantidad de Justi- } \\
\text { ficaciones }\end{array}$ & $\begin{array}{l}\text { Evalúa el número de justificaciones o razones dadas para } \\
\text { soportar un punto de vista. }\end{array}$ \\
\hline
\end{tabular}




\section{0 - Cogency, Journal of reasoning and argumentation}

\begin{tabular}{|c|c|c|c|c|}
\hline & & & & Evalúa presencia/ausencia de diversos puntos de vista. \\
\hline & & $\begin{array}{l}\text { Evalúan movi- } \\
\text { mientos orien- }\end{array}$ & Pluralismo & $\begin{array}{l}\text { Evalúa presencia/ausencia de argumentos con anticipa- } \\
\text { ciones de contraargumentos. }\end{array}$ \\
\hline & $\stackrel{0}{x}$ & $\begin{array}{l}\text { sus propios } \\
\text { puntos de vista, }\end{array}$ & & Evalúa si se mantiene o no la postura defendida. \\
\hline & & $\begin{array}{l}\text { bases y límites } \\
\text { de este (Carre- } \\
\text { her, 1983; Lei- } \\
\text { tão, 2007). }\end{array}$ & Flexibilización P.V. & $\begin{array}{l}\text { Evalúa la incorporación de elementos de oposición al pro- } \\
\text { pio punto de vista. }\end{array}$ \\
\hline & & & & Evalúa la claridad en el diálogo argumentativo. \\
\hline & & & & Evalúa la cohesión en el diálogo argumentativo. \\
\hline & & & & Evalúa la coherencia en el diálogo argumentativo. \\
\hline & & & & $\begin{array}{l}\text { Evalúa la presencia de digresión en el diálogo argumen- } \\
\text { tativo. }\end{array}$ \\
\hline & & & Discursivos & $\begin{array}{l}\text { Evalúa la acción de retomar el argumento/contraargu- } \\
\text { mento. }\end{array}$ \\
\hline & & & & Evalúa la organización del discurso. \\
\hline 足 & & & & Evalúa la presencia de discurso concreto o preciso. \\
\hline$\sum_{\substack{0 \\
0}}$ & & & & Evalúa el uso de ideas mencionadas superficialmente. \\
\hline & & Fvalúan la & & Evalúa la presencia de sesgo en el discurso. \\
\hline & $\stackrel{\Omega}{u}$ & $\begin{array}{l}\text { Stuacion dis- } \\
\text { cursiva y el con- }\end{array}$ & & Evalúa la fluidez en el diálogo argumentativo. \\
\hline & 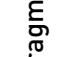 & nicación crítica & & Evalúa la seguridad para expresar las ideas. \\
\hline & & $\begin{array}{l}\text { y Grootendorst, } \\
\text { 2004). }\end{array}$ & & Evalúa el uso de lenguaje acorde a la audiencia. \\
\hline & & & & $\begin{array}{l}\text { Evalúa la actitud del argumentador (expresiones corpo- } \\
\text { rales, etc.). }\end{array}$ \\
\hline & & & & $\begin{array}{l}\text { Evalúa el momento en que un argumento es introducido } \\
\text { en el discurso (pertinencia discursiva). }\end{array}$ \\
\hline & & & Retóricos & Evalúa el tono de voz usado. \\
\hline & & & & Evalúa el estilo de redacción. \\
\hline & & & & Evalúa el uso de discurso persuasivo. \\
\hline & & & & Evalúa la acción de atacar al opositor. \\
\hline & & & & $\begin{array}{l}\text { Evalúa la tendenciosidad en la formulación de las pre- } \\
\text { guntas. }\end{array}$ \\
\hline & & & & Evalúa la reiteración de información. \\
\hline
\end{tabular}




\begin{tabular}{|c|c|c|c|c|}
\hline \multirow{9}{*}{ 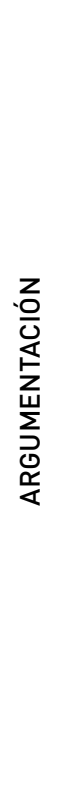 } & \multirow{9}{*}{ 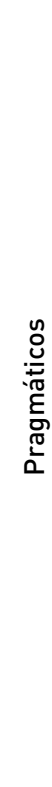 } & \multirow{9}{*}{$\begin{array}{l}\text { Evalúan la } \\
\text { situación dis- } \\
\text { cursiva y el con- } \\
\text { texto de comu- } \\
\text { nicación crítica } \\
\text { (Van Eemeren } \\
\text { y Grootendorst, } \\
\text { 2004). }\end{array}$} & \multirow{9}{*}{$\begin{array}{l}\text { Condiciones de } \\
\text { producción }\end{array}$} & Evalúa el uso del tiempo. \\
\hline & & & & Evalúa la participación de los debatientes. \\
\hline & & & & $\begin{array}{l}\text { Evalúa el respeto por las funciones establecidas en las } \\
\text { fases del debate. }\end{array}$ \\
\hline & & & & Evalúa la organización del equipo debatiente. \\
\hline & & & & Evalúa el trabajo en equipo. \\
\hline & & & & $\begin{array}{l}\text { Evalúa de forma explícita aspectos que caracterizan el } \\
\text { contexto. }\end{array}$ \\
\hline & & & & Evalúa la preparación previa del debate. \\
\hline & & & & $\begin{array}{l}\text { Evalúa la Conclusión pertinente (e.g., si introduce o no } \\
\text { más argumentos). }\end{array}$ \\
\hline & & & & Evalúa el número de intervenciones para hablar. \\
\hline
\end{tabular}

Nota. Latorre (2018); Ramírez et al. (2013); Ramírez (2012).

Finalmente, el macroanálisis permitió identificar las transformaciones en el uso de criterios por sujeto, las regularidades, las comunalidades y las diferencias encontradas en los casos seleccionados de los dos países.

\section{Resultados}

Los resultados privilegian las producciones discursivas producidas en contexto de aula, las cuales se muestran en función de los momentos de análisis y los objetivos del artículo. De este modo, en primer lugar, se muestra cómo se realizó la identificación y análisis de los indicadores en las evaluaciones de los estudiantes a partir de las categorías mencionadas en el procedimiento analítico; en segundo lugar, se responde al objetivo de transformación en el uso de criterios a nivel intra e interindividual; por último, son analizadas las comunalidades y diferencias existentes entre los dos países.

\section{Identificación y análisis de criterios de evaluación}

Esta identificación se da a partir del microanálisis realizado a las 36 evaluaciones producidas por los diez casos analizados. En la tabla 3, se hace visible los criterios e indicadores de evaluación del argumento - dialécticos y estructurales, y de la argumentación - reflexivos, discursivos, retóricos y condiciones de producción -, utilizados en cada debate por los participantes de cada país. 
52 - Cogency, Journal of reasoning and argumentation

Tabla 3.

Frecuencia de criterios usados por los participantes en los debates de Brasil y Colombia

\begin{tabular}{|c|c|c|c|c|c|c|c|c|c|c|c|c|}
\hline \multirow{2}{*}{\multicolumn{2}{|c|}{ Criterio }} & \multirow{2}{*}{ Indicador } & \multicolumn{5}{|c|}{ Brasil } & \multicolumn{4}{|c|}{ Colombia } & \multirow{2}{*}{$\begin{array}{l}\text { Total } \\
\text { fr. }\end{array}$} \\
\hline & & & D1 & D2 & D3 & D4 & D5 & D1 & D2 & D3 & D4 & \\
\hline \multirow{14}{*}{ 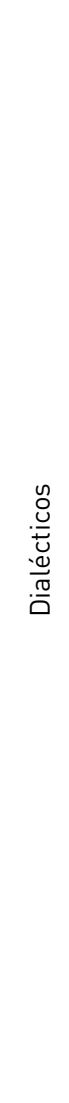 } & \multirow{12}{*}{ Aceptabilidad } & Autoridad fuente & 3 & 1 & & 2 & 1 & & & & & 7 \\
\hline & & Cantidad referencias & & & & & & & & 2 & 3 & 5 \\
\hline & & $\begin{array}{l}\text { Consistencia tipo de } \\
\text { información usada como } \\
\text { apoyo P.V. }\end{array}$ & 1 & & & & & 1 & & & & 1 \\
\hline & & Critica analogía & & & & & & 1 & & & & 1 \\
\hline & & Falta fundamentos & 1 & & 2 & 1 & 3 & & & & & 7 \\
\hline & & Plausibilidad & & & & & & 1 & & & & 1 \\
\hline & & Uso conceptos técnicos & & & & & & & 2 & & & 2 \\
\hline & & Uso ejemplos & & & & & 5 & & & & & 5 \\
\hline & & Uso evidencia científica & 6 & 1 & 6 & 7 & 8 & & 1 & & & 31 \\
\hline & & $\begin{array}{l}\text { Uso información hipo- } \\
\text { tética }\end{array}$ & & & & 1 & & & & & & 1 \\
\hline & & $\begin{array}{l}\text { Uso información sentido } \\
\text { común }\end{array}$ & 2 & 1 & 1 & 2 & 2 & & & & & 8 \\
\hline & & $\begin{array}{l}\text { Uso referencias con- } \\
\text { fiables }\end{array}$ & 4 & 5 & 1 & 5 & 3 & & 5 & 22 & 8 & 53 \\
\hline & Relevancia & $\begin{array}{l}\text { Relación entre justifica- } \\
\text { ción (J) y P.V. }\end{array}$ & & & & & & 1 & 5 & & 1 & 7 \\
\hline & Suficiencia & $\begin{array}{l}\text { Necesidad de presentar } \\
\text { más J para dar mayor } \\
\text { soporte al P.V. }\end{array}$ & & & & & & & 5 & & & 5 \\
\hline \multirow{2}{*}{ 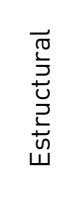 } & $\begin{array}{l}\text { Cantidad de } \\
\text { Justificacio- } \\
\text { nes }\end{array}$ & $\begin{array}{l}\text { Cantidad de Justifica- } \\
\text { ciones }\end{array}$ & 2 & 6 & 4 & 0 & 2 & 6 & & 15 & 5 & 40 \\
\hline & $\begin{array}{l}\text { Elementos } \\
\text { Arg. }\end{array}$ & Presencia de P.V. + J. & & & 1 & & 1 & & & & 1 & 3 \\
\hline \multirow{2}{*}{ 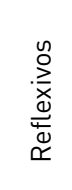 } & \multirow{2}{*}{$\begin{array}{l}\text { Flexibiliza- } \\
\text { ción P.V. }\end{array}$} & $\begin{array}{l}\text { Mantener postura de- } \\
\text { fendida }\end{array}$ & & 1 & & & 1 & 3 & 1 & & & 6 \\
\hline & & $\begin{array}{l}\text { Incorporación elemen- } \\
\text { tos oposición P.V. }\end{array}$ & & & & 1 & 1 & & & & 1 & 3 \\
\hline \multirow{7}{*}{ 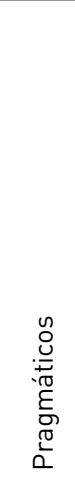 } & \multirow{7}{*}{ Discursivos } & Claridad & 1 & 4 & 2 & 2 & 3 & 5 & 17 & 26 & 9 & 69 \\
\hline & & Cohesión & & & & & & & 1 & 2 & 1 & 4 \\
\hline & & Digresión & 1 & & & & & 1 & 4 & 3 & 5 & 14 \\
\hline & & Discurso Concreto & & & & & & 1 & 4 & 2 & 2 & 7 \\
\hline & & Organización Discurso & & & & & & & 3 & & & 5 \\
\hline & & $\begin{array}{l}\text { Redundancia de argu- } \\
\text { mentos }\end{array}$ & & 1 & & 1 & & & & & & 2 \\
\hline & & $\begin{array}{l}\text { Retomar el argumento / } \\
\text { contraargumento }\end{array}$ & 3 & 6 & 5 & 8 & 11 & 1 & 7 & 16 & 3 & 60 \\
\hline
\end{tabular}




\begin{tabular}{|c|c|c|c|c|c|c|c|c|c|c|c|c|}
\hline \multirow{12}{*}{ 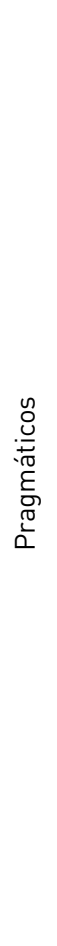 } & \multirow{5}{*}{ Retóricos } & Actitud del argumentador & & & & & & & 2 & & & 2 \\
\hline & & $\begin{array}{l}\text { Actuación coherente con } \\
\text { papel }\end{array}$ & 2 & 2 & 2 & 2 & & & & & & 8 \\
\hline & & Discurso Persuasivo & & & & & & 1 & & & & 1 \\
\hline & & $\begin{array}{l}\text { Seguridad expresión } \\
\text { ideas }\end{array}$ & 2 & 3 & 5 & 3 & 2 & 5 & 1 & 1 & 3 & 25 \\
\hline & & Sesgo pregunta & & & & & & & & 1 & & 1 \\
\hline & \multirow{7}{*}{$\begin{array}{l}\text { Condiciones } \\
\text { de produc- } \\
\text { ción }\end{array}$} & Ausencia conclusión & 1 & & 1 & & & & & & & 2 \\
\hline & & $\begin{array}{l}\text { Coherencia entre la } \\
\text { pregunta y el papel des- } \\
\text { empeñado }\end{array}$ & 1 & 2 & & & & & & & & 3 \\
\hline & & $\begin{array}{l}\text { Coherencia de la res- } \\
\text { puesta dada con la } \\
\text { pregunta }\end{array}$ & & 3 & 1 & 3 & 3 & & & & & 10 \\
\hline & & Conclusión pertinente & & 2 & & & 2 & & & & 3 & 6 \\
\hline & & $\begin{array}{l}\text { Cumplimiento función } \\
\text { debate }\end{array}$ & 1 & 2 & 2 & 3 & 1 & & & & & 9 \\
\hline & & Participación & & & & & & & 4 & 4 & 2 & 10 \\
\hline & & Uso de Tiempo & & & & & & & 7 & 21 & 10 & 38 \\
\hline
\end{tabular}

Nota. Fuente propia.

Con respecto al uso de criterios dialécticos, la evaluación de los estudiantes se direcciona al examen de la aceptabilidad del argumento en cuanto al grado de plausibilidad de las informaciones usadas, según la confiabilidad y la naturaleza epistémica la información ofrecida como apoyo al punto de vista (estadística, experiencial, científica, subjetiva), y el prestigio de la fuente, indicadores que permiten confirmar si una justificación es o no 'una buena razón' para soportar el punto de vista defendido.

Los indicadores que más frecuencias de uso tienen son, 'referencias confiables' (f. 53) y 'evidencia científica' (f. 31). Los criterios estructurales direccionados a evaluar los elementos del argumento en sí fueron usados en menor proporción, siendo el indicador cantidad de justificaciones el más frecuente (f. 40).

Con respecto al examen de la argumentación predomina el uso de criterios pragmáticos direccionados a valorar el discurso, la retórica y las condiciones de producción. El foco de análisis de los indicadores que aquí emergen juzga la situación discursiva como un todo - el contexto de comunicación crítica propuesto por el design pedagógico - que hace posible que la argumentación sea de hecho creada y sustentada. Los indicadores discursivos que más predominan se relacionan con la 'claridad del discurso' (f. 69) y la acción de 'retomar los argumentos del otro' (f. 60) cuando se instaura la contraargumentación y se concluye el debate. Los aspectos retóricos que fueron más evaluados se relacionan con la 'seguridad del argumentador para expresar sus ideas' (f. 25) y la 'coherencia del papel desempeñado durante 
el debate' (f. 8). En cuanto a las condiciones de producción hay un predominio de indicadores frente al 'cumplimiento de la función', en las que el 'uso del tiempo' (f. 38), la 'participación' (f. 10) y la 'coherencia de la respuesta con la pregunta' (f. 10) adquieren mayor relevancia en las valoraciones realizadas por los estudiantes.

Así mismo, y en menor medida, aparece la utilización de criterios reflexivos cuyo fin es reconocer y valorar en los argumentadores la flexibilidad (f. 9) y diversidad de puntos de vista al momento de ser establecida la discusión crítica.

A nivel general, se concluye que hay mayor diversidad y dispersión de indicadores pragmáticos que dialécticos lo que muestra que, las valoraciones de los participantes se centran con mayor preponderancia en la situación discursiva del debate crítico.

Transformación en el uso de criterios inter e intraindividual

El análisis de transformaciones a nivel intersujeto con respecto al uso de criterios de evaluación de la calidad, para los dos países, permite evidenciar variabilidad tanto en el momento del aparecimiento de los criterios como en su frecuencia de uso.

En la Tabla 4, se observa que los criterios dialécticos, estructurales, reflexivos, discursivos y retóricos son introducidos por la mayoría de los estudiantes desde el D1, se exceptúa de esta regla el criterio condiciones de producción, el cual aparece en el D2. Con respecto a la frecuencia de uso, los criterios dialécticos son presentes con mayor frecuencia en el D3 y D4, los estructurales la mayoría lo utilizan en el D3, los reflexivos son poco usados, pero aparecen en los D1, D2, D4 y D5. Los discursivos son usados en todos los debates con alta frecuencia, especialmente, en el D3; los retóricos también son empleados en todos los debates, pero con menor frecuencia que los discursivos; por su parte el criterio condiciones de producción tienen un uso más frecuente a partir del D2, y prevalecen más que los retóricos.

Como conclusión general, la mayor frecuencia de uso de todos los criterios se da en el D3. El uso de criterios de calidad de las evaluaciones realizadas, por estudiantes de Brasil y Colombia, de las argumentaciones de sus compañeros, muestra que, los criterios pragmáticos de tipo discursivo y dialécticos (aceptabilidad) son los de mayor utilización, sin embargo, no siempre los sujetos usaron los criterios en todos los debates y con la misma frecuencia. 
Tabla 4.

Uso de criterios por participante y debate Brasil y Colombia

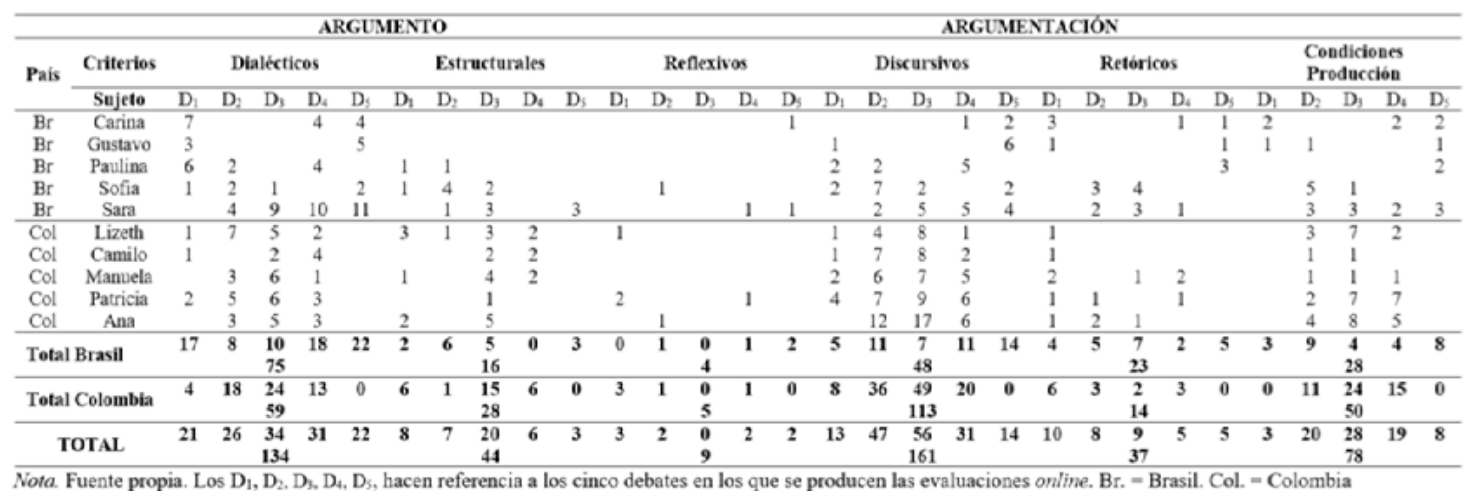

A nivel intrasujeto se observa que la mayoría de los estudiantes introducen en sus valoraciones criterios dialécticos, estructurales, discursivos y retóricos desde el debate uno, sin embargo, existe variabilidad en los indicadores que cada participante utiliza debate a debate. Para ejemplificar lo anterior, en la Tabla 5 se presentan las transformaciones en el uso de indicadores de evaluación de los casos de Sara (Brasil) y Lizeth (Colombia), quienes fueron escogidos por ser en los que se encontró mayor diversidad de criterios.

\section{Tabla 5.}

Ejemplos de uso de indicadores intrasujeto

\begin{tabular}{|c|c|c|c|c|c|c|c|c|c|c|}
\hline \multirow{2}{*}{ Criterio } & \multirow{2}{*}{ Indicador } & \multicolumn{5}{|c|}{ Sara } & \multicolumn{4}{|c|}{ Lizeth } \\
\hline & & D1 & D2 & D3 & D4 & D5 & D1 & D2 & D3 & D4 \\
\hline \multirow{12}{*}{ Aceptabilidad } & Autoridad fuente & & 1 & & & 1 & & & & \\
\hline & Cantidad referencias & & & & & & & & & \\
\hline & $\begin{array}{l}\text { Consistencia tipo de información } \\
\text { usada como apoyo al punto de vista } \\
\text { (P.V.) }\end{array}$ & & & & & & & & & \\
\hline & Critica analogía & & & & & & 1 & & & \\
\hline & Falta fundamentos & & & 2 & & 3 & & & & \\
\hline & Plausibilidad & & & & & & & & & \\
\hline & Uso conceptos técnicos & & & & & & & & & \\
\hline & Uso ejemplos & & & & & 2 & & & & \\
\hline & Uso evidencia científica & & & 6 & 4 & 1 & & & 1 & \\
\hline & Uso información hipotética & & & & 1 & & & & & \\
\hline & Uso información sentido común & & & & 1 & 2 & & & & \\
\hline & Uso referencias confiables & & 3 & 1 & 4 & 2 & & 1 & 4 & 2 \\
\hline Relevancia & Relación entre justificación (J.) y P.V. & & & & & & & 3 & & \\
\hline
\end{tabular}




\begin{tabular}{|c|c|c|c|c|c|c|c|c|c|}
\hline Suficiencia & $\begin{array}{l}\text { Necesidad de presentar más J. para } \\
\text { dar mayor soporte al P.V. }\end{array}$ & & & & & & 3 & & \\
\hline $\begin{array}{l}\text { Cantidad de Jus- } \\
\text { tificaciones }\end{array}$ & Cantidad de Justificaciones & 1 & 2 & & 2 & 2 & & 3 & 2 \\
\hline $\begin{array}{l}\text { Elementos Argu- } \\
\text { mento }\end{array}$ & Presencia de P.V. más J. & & 1 & & 1 & 1 & & & \\
\hline \multirow{2}{*}{$\begin{array}{l}\text { Flexibilización } \\
\text { P.V. }\end{array}$} & Mantener postura defendida & & & & 1 & 1 & & & \\
\hline & $\begin{array}{l}\text { Incorporación elementos oposición } \\
\text { P.V. }\end{array}$ & & & 1 & & & & & \\
\hline \multirow[t]{4}{*}{ Discursivos } & Claridad & & 2 & & 1 & & 2 & 6 & \\
\hline & Cohesión & & & & & & 1 & & \\
\hline & Digresión & & & & & & & 1 & \\
\hline & Retomar & 2 & 3 & 5 & 3 & 1 & 1 & 1 & 1 \\
\hline \multirow[t]{2}{*}{ Retóricos } & Actuación coherente con papel & 1 & 1 & & & & & & \\
\hline & Seguridad expresión ideas & 1 & 2 & 1 & & 1 & & & \\
\hline \multirow{6}{*}{$\begin{array}{l}\text { Condiciones de } \\
\text { producción }\end{array}$} & Coherencia pregunta con papel & 1 & & & & & & & \\
\hline & Coherencia respuesta dada pregunta & 1 & 1 & 1 & 2 & & & & \\
\hline & Conclusión pertinente & & & & 1 & & & & \\
\hline & Cumplimiento función debate & 1 & 2 & 1 & & & & & \\
\hline & Participación & & & & & & 2 & 1 & \\
\hline & Uso de Tiempo & & & & & & 1 & 6 & 2 \\
\hline
\end{tabular}

Nota. Fuente propia. Ejemplos correspondientes al corpus de análisis construido por Ramírez (2012) y Latorre (2018).

Como se observa en la Tabla 5 Lizeth y Sara hacen un mayor uso de indicadores dialécticos, especialmente, 'referencias confiables', sin embargo, Lizeth no solo utiliza indicadores de 'aceptabilidad', sino que incluye indicadores para evaluar la 'relevancia' y 'suficiencia del argumento', mientras que Sara se centra en la valoración de la 'aceptabilidad' de las justificaciones haciendo un uso más diversificado de esta, por ejemplo, indicadores de 'información sentido común,' 'evidencia científica' y 'uso de ejemplos', entre otros.

El indicador más usado del criterio estructural por las dos participantes 'cantidad de justificaciones', en el caso de Lizeth este indicador se utiliza con mayor frecuencia. Los criterios 'reflexivos', también aparecen en ambos casos, pero con baja frecuencia de uso y en pocos debates. Los criterios discursivos son introducidos por Sara en mayor frecuencia desde el D2 y en Lizeth desde el D1. El uso más diversificado de este criterio fue realizado por Lizeth. En ambos casos, el indicador que más se usa para evaluar todos los debates es 'retomar argumentos del otro'. Los criterios retóricos aparecen en ambos casos, pero Sara le da mayor importancia a este criterio usando un mayor número de indicadores y presentando mayor frecuencia de uso. Finalmente, en el criterio condiciones de producción Sara diversifica su juicio a través un mayor 
uso de indicadores en comparación con Lizeth. En cuanto al uso de estos indicadores, es importante destacar que Sara evalúa las argumentaciones de sus pares en función de las condiciones discursivas y comunicativas del debate crítico, mientras que Lizeth centra su juicio de valor en el 'uso del tiempo' y en la 'participación de todos los debatientes' como regla implícita en el contexto de la clase.

\section{Comunalidades y Diferencias en el uso de Criterios - Brasil y Colombia}

Cualitativamente los casos analizados, de Brasil y Colombia, utilizan en sus evaluaciones las diferentes categorías propuestas por Latorre (2018); Ramírez et al. (2013) y Ramírez (2012), de modo que, a nivel general, en la Figura 1 se observan comunalidades y diferencias en los criterios e indicador de criterio en cuanto a, presencia/ausencia, frecuencia de uso, y reiteración en la utilización durante los debates examinados.

\section{Figura 1.}

Diferencias en el uso indicadores de la calidad de la evaluación entre Brasil y Colombia

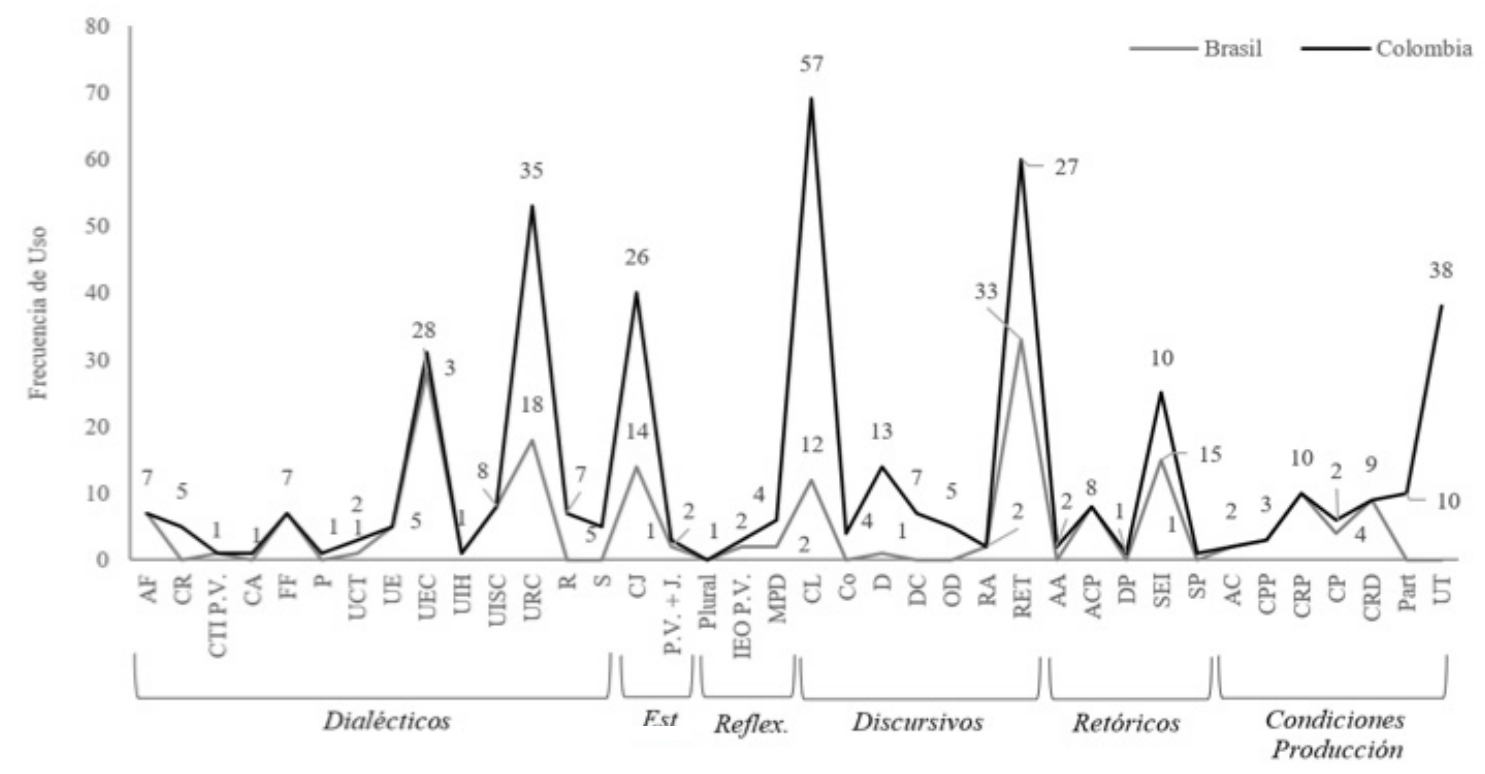

Nota. $\mathrm{AF}=$ Autoridad de la fuente. $\mathrm{CR}=$ Cantidad de referencias. CTI P.V. = Coherencia tipo de información con el punto de vista. $\mathrm{CA}=$ Crítica a la analogía. FF = Falta de fundamentos. $\mathrm{P}=$ Plausibilidad. UCT = Uso conceptos técnicos. UE = Uso Ejemplos. UEC = Uso evidencia científica. UIH = Uso información hipotética. UISC = Uso información sentido común; URC = Uso referencias confiables. $\mathrm{R}=$ Relevancia; $\mathrm{S}=$ Suficiencia. $\mathrm{CJ}=\mathrm{Cantidad}$ justificaciones. P.V.+J. = Punto de vista más justificación. P= Pluralidad. IEO P.V.= Integración elementos de la oposición al punto de vista. MPD = Mantenimiento de la posición defendida. $\mathrm{Cl}=\mathrm{Claridad}$. $\mathrm{Co}=\mathrm{Cohesión}$. D = Digresión. $\mathrm{DC}=$ Discurso concreto. $\mathrm{OD}=$ Organización discurso. RA= Redundancia de argumentos. RET = Retomar. $\mathrm{AA}=$ Actitud Argumentador. $\mathrm{ACP}=$ Actuación coherente con papel. DP = Discurso Persuasivo; SEI $=$ Seguridad expresión ideas. $\mathrm{SP}=$ Sesgo pregunta. $\mathrm{AC}=$ Ausencia de conclusión. $\mathrm{CPP}=$ Coherencia pregunta con papel. $\mathrm{CRP}=$ Coherencia respuesta-pregunta. $\mathrm{CP}=$ Conclusión pertinente. $\mathrm{CRD}=$ Cumplimiento función debate. Part $=$ Participación. UT $=$ Uso Tiempo. Est $=$ Estructurales. Reflex $=$ Reflexivos. 
Las comunalidades más notorias entre los dos países se dan a nivel general, en el criterio reflexivo, que a pesar de tener baja frecuencia de uso aparece en los debates $\mathrm{y}$, a nivel de indicadores de criterio en la frecuencia y reiteración de uso en cuanto a 'retomar argumentos' y 'seguridad en la expresión de las ideas'. Análisis que refleja un patrón valorativo centrado en la práctica argumentativa y que evidencia el impacto inicial que pudo tener el design pedagógico sobre la apropiación de estos indicadores en el proceso evaluativo. Es importante aclarar que la frecuencia de uso de los indicadores reflexivos sería un indicio de incorporación bajo, por tanto, estos debem ser entrenados con mayor tiempo e intensidad.

Las diferencias entre los países se dan frente a la mayor o menor frecuencia de uso de indicador de criterio. A nivel dialéctico los participantes de Brasil le otorgan mayor valor a la 'autoridad de la fuente', la 'cantidad de referencias', la falta de fundamentos', el 'uso de ejemplos', el uso de 'evidencia científica', el uso de 'información de sentido común', mientras que, los participantes de Colombia privilegian el uso de 'referencias confiables', la 'relevancia', y la 'suficiencia'. Hallazgos que indican que en los casos brasileros el examen de los argumentos producidos por otros se centra en la aceptabilidad de las justificaciones y en los casos colombianos involucra, además, la relevancia y la suficiencia. Cabe aclarar que estos dos últimos criterios se presentan en dos de los casos analizados asociados a la introducción de estas nociones en el aula de clase.

A nivel estructural, los resultados muestran que el indicador de criterio 'cantidad de justificaciones se presenta en los dos países, sin embargo, en Colombia se hace un mayor uso de este. A nivel discursivo, la mayoría de los indicadores (claridad, cohesión, digresión, discurso concreto, organización discurso) son usados con mayor frecuencia por los participantes de Colombia, mientras que, los casos brasileños privilegian los indicadores retóricos (actuación coherente con el papel).

Finalmente, en cuanto al criterio condiciones de producción las diferencias se marcan por la presencia/ausencia de indicadores en alguno de los dos países, por ejemplo, en Brasil se privilegia la 'coherencia entre la respuesta y la pregunta' y el 'cumplimiento de funciones en las fases del debate', y los casos colombianos le dan importancia a la 'participación de todos los integrantes de cada equipo en el debate' y al respeto por el 'uso del tiempo'.

\section{Discusión}

El objetivo de este estudio fue comparar la utilización que estudiantes universitarios, de Brasil y Colombia, involucrados en un escenario argumentativo, realizan de criterios de calidad del argumento y de la argumentación. Para esto, se discute sobre 
las diferencias y comunalidades en el uso de los indicadores analíticos, así como, las transformaciones en la competencia para evaluar online debates críticos.

\section{Comunalidades y diferencias entre países}

Los resultados presentados en este estudio evidencian que, al comparar los casos de los dos países, se encuentra la presencia de comunalidades y diferencias en el uso de indicadores de evaluación de la calidad del argumento y de la argumentación. Por ejemplo, se observan diferencias en cuanto a la valoración de criterios pragmático-discursivos y de condiciones de producción, siendo más relevantes en el contexto colombiano que en el brasileño; por el contrario, en el contexto brasileño se observa preponderancia en la utilización de criterios orientados a examinar la plausibilidad de las justificaciones, es decir, mayor presencia del criterio dialéctico-aceptabilidad. Estas diferencias y semejanzas pueden estar asociadas a características culturales, a las metodologías y los mediadores propios del design implementado en cada país y a la complejidad de los procesos cognitivos involucrados en la evaluación.

La explicación sobre las características culturales se centra en la comprensión de las nociones de desarrollo cognitivo, en las que existe una interrelación entre aspectos contextuales (política nacional, valores institucionales, prácticas curriculares, políticas educativas y prácticas interpersonales) y procesos individuales de internalización (creencias personales), a través de los cuales los sujetos incorporan normas y valores culturales, y se median formas de funcionamiento asociadas a prácticas cotidianas (Rogoff, 2003; Super y Harkness, 2002; Wang y Brockmeier, 2002). En este sentido, el proceso de evaluación de argumentos y argumentaciones esta atravesado por los conocimientos previos, las creencias, los valores culturalmente construidos, los cuales pueden flexibilizar o no, tanto el examen que se hace sobre los propios puntos de vista, como de los puntos de vista ofrecidos por los otros (Walton, 1992; 2010). Comprensión que se refleja en los focos evaluativos que predominaron en cada país.

Es relevante mencionar que, a pesar de que se mantienen invariantes estructurales en el design pedagógico de los dos países, una posible explicación de las diferencias y comunalidades encontradas se relaciona con las adecuaciones asociadas a prácticas y características curriculares propias de cada contexto, ya que consideramos que el proceso de aprendizaje esta mediado por el papel que ejerce el lenguaje, como constitutivo del pensamiento y las interacciones sociales tanto en la construcción del conocimiento como en el desarrollo de competencias argumentativas (Andrew, 2010; Müller-Mirza y Perret-Clermont, 2009; Valsiner, 2006; Vygotski, 2000/1934). Por ejemplo, en cuanto a los mediadores, en Brasil fueron estudiantes de nivel de maestría con mayor dominio en argumentación, mientras que en Colombia fueron 
estudiantes de último semestre entrenados para la implementación del desing pedagógico. Al respecto de las características curriculares como, ubicación en el semestre, naturaleza de la asignatura e intensidad horaria, en Brasil se dio en el primer semestre, como curso obligatorio del currículo y con mayor intensidad horaria, mientras que en Colombia se ofreció en tercer semestre como materia electiva y menor intensidad horaria. En este caso, los estudiantes de tercer semestre ya tienen algún dominio sobre el contenido psicológico debatido.

En el conjunto de casos analizados emerge el uso de indicadores relacionados con la flexibilización del punto de vista (e.g., mantener la postura defendida, incorporación de elementos de oposición al punto de vista) y el uso de criterios dialécticos de relevancia y de suficiencia. Sin embargo, su utilización es poco frecuente coincidiendo con la introducción de los criterios de calidad propuestos por Govier (2010), la función evaluativa desempeñada en el debate, y con la apropiación del discurso sobre argumentación usado en la disciplina.

Aunque, conceptos sobre teoría de argumentación fueron trabajados, el tiempo de estudio, tal vez, fue insuficiente para generar aprendizaje y práctica para una mayor apropiación. Asimismo, el grado de complejidad conceptual inherente a tales criterios (Larson et al., 2009), requiere un mayor nivel de análisis y evaluación de la información ofrecida en el contexto discursivo-argumentativo en el que están inmersos. Probablemente, el nivel de sofisticación esperado debería responder a la cantidad y complejidad de los objetivos de aprendizaje que la disciplina pretendía alcanzar y el tiempo que llevó alcanzar estos objetivos (Müller-Mirza y Perret-Clermont, 2009).

En ambos países prima el uso de criterios para evaluar la argumentación (pragmáticos) y el argumento (dialécticos-aceptabilidad). La presencia de indicadores pragmáticos se puede explicar, por un lado, como resultado de la participación de los sujetos en diversas situaciones evaluativas, no solo de carácter argumentativo, y por otro lado, el objetivo perseguido cuando se involucran en situaciones argumentativas no se centra solamente en la resolución crítica de la diferencia de opinión (Van Eemeren et al., 2008; Gaviria y Corredor, 2011). Esta comprensión se apoya en la noción de desarrollo cognitivo desde las perspectivas socioculturales, a partir de las cuales los valores, las normas, y creencias direccionan y regulan el funcionamiento psicológico intrapersonal e interpersonal (Bruner, 1990; Rogoff, 2003; Super y Harkness, 2002; Valsiner, 2001; Wang y Brockmeier, 2002; Wertsch, 2008/1979), aspectos que son transferidos a la situación argumentativa, por ejemplo, el debate, noción que en los contextos analizados, generalmente, se asume con una carga negativa (convencer a cualquier costo que mi punto de vista es verdadero, reafirmar mi punto de vista), por encima de la racionalidad de las justificaciones ofrecidas. 
La alta frecuencia de uso de los criterios de 'aceptabilidad' puede atribuirse a la participación de los estudiantes en la estrategia pedagógica, a través del contenido sobre argumentación impartido en la asignatura acerca de las distinciones entre opinión/opinión razonada y tipos de información. Se entiende que en el contexto de aula se le da gran importancia a la naturaleza epistemológica de la información ofrecida como soporte para el punto de vista (Barchfeld y Sodian, 2009; Clark y Sampson, 2008; Gaviria y Corredor, 2011; Glassner et al., 2005; Govier, 2010; Kuhn y Franklin, 2006; Kuhn y Pearsall, 2000), siendo válido aceptar argumentos basados en recursos de autoridad (investigación, expertos, evidencia), en lugar de argumentos cuyos fundamentos son del tipo de experiencia personal o información subjetiva, elemento que explica la mayor frecuencia de uso de 'referencias confiables', 'evidencia científica'.

Por su parte, el bajo uso de criterios reflexivos y dialécticos (relevancia, suficiencia) puede comprenderse por la mayor complejidad cognitiva que involucra la capacidad de reconocer en otros participantes habilidades como la revisión de perspectivas, la incorporación de elementos del contraargumento al propio punto de vista, así como, la exigencia en el examen de la solidez de las afirmaciones producidas de forma oral en un contexto de debate crítico. Otra posible explicación es la introducción tardía de estos criterios y, en consecuencia, el poco tiempo para su entrenamiento.

Transformaciones en el uso de criterios

Existen variaciones en el uso de criterios de evaluación para examinar la calidad de los argumentos y de la argumentación ofrecida por otros, en un contexto altamente argumentativo. Dichas variaciones se observan, en alguna medida, cuando los estudiantes realizan sus evaluaciones de cada debate, a pesar de que, los indicadores no sean incorporados de la misma manera por todos los casos analizados.

Una explicación a estas variaciones es el proceso de incorporación de criterios en las evaluaciones, los cuales se van volviendo más diversificados y minuciosos a media que se va progresando en los contenidos y en la práctica argumentativa en contexto de aula. Esta variación no implica, necesariamente, que los indicadores previamente construidos se dejen de lado o sean acumulativos, sino que, el foco de evaluación cambia de acuerdo con las exigencias de la situación y los intereses del evaluador. Por ejemplo, algunas veces se da prioridad a cuestiones más normativas que a la estructura interna del argumento (uso de criterios de aceptabilidad) o se privilegia la situación discursiva de debate. Estas variaciones en el proceso de incorporación, diversificación y sistematización de los criterios de sujeto a sujeto 
durante el tiempo de la intervención, tal vez, se deba a la influencia de factores internos (e.g., motivación, atención, autoeficacia), como externos (e.g., complejidad del contenido, tiempo de práctica, nivel de apropiación de los contenidos), que hacen con que los sujetos adopten nuevas formas de significación (Clark y Sampson, 2008; Rennau, 2004).

\section{Conclusiones}

Este estudio contribuye a ampliar la comprensión de la complejidad del proceso evaluativo en contexto educativos auténticos y en casos de diferentes países, corroborando el mapeo de criterios e indicadores que involucran tanto el argumento como la práctica argumentativa y al argumentador, a diferencia de los estudios presentes en la literatura los cuales hacen análisis de la evaluación centrándose en la estructura del argumento, la validez de las justificaciones y la relación que existe entre el punto de vista y las razones que lo sustentan (Gaviria y Corredor, 2011; Govier, 2014; Kuhn, 1991; Kuhn y Udell, 2003; Van Eemeren et al., 2008).

Este estudio coincide con los hallazgos sobre la dificultad en la utilización de criterios para evaluar la relación que existe entre el punto de vista y las razones que lo sustentan, así como la suficiencia de estas (Barchfeld y Sodian, 2009; Felton y Kuhn, 2001; Gaviria y Corredor, 2011; Kuhn y Pearsall, 2000; Larson et al., 2009; ). También, fue evidente la dificultad en el uso de criterios reflexivos - revisión de perspectivas y la flexibilidad en los posicionamientos -, a pesar de ser privilegiados en el contexto del design pedagógico. Por tanto, se considera que las planeaciones de aula no sólo deben incluir acciones educativas focalizadas a generar mayor apropiación y uso de criterios de calidad del argumento - ARS y de criterios reflexivos, sino también, pensar en intervenciones que involucren la competencia evaluativa por más tiempo y con mayor intensidad.

Para futuras investigaciones, se recomienda ampliar el corpus de análisis en cuanto a la cantidad (número de casos), y a la diversificación de situaciones en las que los estudiantes evalúan los argumentos y argumentaciones de sus pares. Asimismo, los criterios mapeados por Latorre (2018); Ramírez et al. (2013) y Ramírez (2012) pueden servir de base para construcción de instrumentos orientados al seguimiento de la competencia evaluativa en diferentes poblaciones. 


\section{Referencias}

Abrami, Philip; Bernard, Robert; Borokhovski, Eugene; Waddington, David; Wade, Anne y Persson, Tonje. "Strategies for Teaching Students to Think Critically: A Meta-Analysis". Review and educational research, XX, X, (2014). pp. 1-40. doi: $10.3102 / 0034654314551063$.

Andrews, Richard. Why Argument? In Andrews, Richard. Argumentation in Higher Education: Improving Practice Through Theory and Research (pp. 1-22). New York: Routledge, 2010.

Baker, Michael. "Argumentation et co-construction des connaissances". Interaction et Cognitions, 2/3(1), (1996): 157-191.

Barchfeld, Petra y Sodian, Beate. "Differentiating Theories from Evidence: The Development of Argument Evaluation Abilities in Adolescence and Early Adulthood". Informal Logic, 29(4), (2009): 396-416. doi: 10.22329/il.v29i4.2906.

Britt, M. Anne; Kurby, Christopher A.; Dandotkar, Srikanth y Wolfe, Christopher R. "I agreed with what? Memory for simple argument claims". Discourse Processes, 45, (2008): 52-84. https://doi.org/10.1080/01638530701739207.

Brown, Anne. "Transforming schools into commmunities of thinking and learning about serious matters". American Psychologist, 52, (1997): 399-413. https://doi. org/10.1037/0003-066X.52.4.399.

Bruner, Jerome. Acts of meaning. Cambridge, MA: Harvard University Press. 1990.

Carraher, David y Schliemann, Ana-Lúcia. “The Transfer Dilemma”. The journal of the learning sciences, 11(1), (2002): 1-24. https://doi.org/10.1207/S15327809JLS1101_1.

Cassiani, Suzani. Linguagens e histórias da ASCT: perspectivas educativas. Foro -Taller Apropiación Social de la Ciencia y la Tecnología. Medellín, Colombia, octubre. 2010.

Causado-Escobar, Robert; Santos-Carrasco, Blanca y Calderón-Salas, Idalí. "Desarrollo del pensamiento crítico en el área de ciencias naturales en una escuela de secundaria”. Rev. Fac. Cienc., 4(2), (2015): 17-42. https://doi.org/10.15446/rev.fac.cienc. $\mathrm{v} 4 \mathrm{n} 2.51437$.

Clark, Douglas B. y Sampson, Victor. "Assessing Dialogic Argumentation in Online Environments to Relate Structure, Grounds, and Conceptual Quality". Journal of research in science teaching, 45(3), (2008): 293-321. doi: 10.1002/tea.20216.

Crowell, Amanda y Kuhn, Deanna. "Developing Dialogic Argumentation Skills: A 3-year Intervention Study". Journal of Cognition and Development, 15:2, (2014): 363-381. http://www.tandfonline.com/doi/abs/10.1080/15248372.2012.725187. 
Fuentes, Claudio. Elementos para o Desenho de um Modelo de Debate Crítico na Escola. En S. Leitão, y M. C. Damianovic, Argumentação na Escola: O Conhecimento em Construção (pp. 225-249). São Paulo: Pontes Editores, 2011.

Gaviria, Christian y Corredor, Javier. "Coordinación de Evidencias, Explicaciones, Objetivos en la Argumentación”. Revista Colombiana de Psicología, 21(1), (2011): 43-56. https://revistas.unal.edu.co/index.php/psicologia/article/view/17749/28673.

Glassner, Amnon; Weinstock, Michael y Neuman, Yair. "Pupils' evaluation and generation of evidence and explanation in argumentation". British Journal of Educational Psychology, 75(1), (2005): 105-118. https://doi.org/10.1348/000709904X22278.

Goldstein, Marion; Crowell, Amanda y Kuhn, Deanna. "What Constitutes Skilled Argumentation and How Does it Develop?" Psychology and Argumentation, 29(4), (2009): 379-395. https://doi.org/10.22329/il.v29i4.2905.

Govier, Trudy. A practical study of argument (10 ed.). Wadsworth: Cengalge Learning, 2014.

Halpern, Diane. Thought and knowledge. An introduction to critical thinking. New York: Psychology Press, 2014.

Hemberger, Laura, Kuhn, Deanna, Matos, Flora y Shi, Yuchen. A dialogic path to evidence-based argumentive writing. Journal of the Learning Sciences 26(4), (2017): 575-607. doi: 10.1080/10508406.2017.1336714.

Jiménez-Aleixandre, Maria Pilar y Erduran, Sibel. Argumentation in Science Education: An Overview. En S. Erduran y M. P. Jiménez-Aleixandre (pp. 3-26). Argumentation in Science Education: Perspectives from Classroom-Based Research. Londom: Springer, 2007.

Instituto Colombiano para la Evaluación de la Educación [ICFES]. Informe Nacional de Resultados examen Saber 11, 2017. Recuperado de https://www.icfes.gov.co/documents/20143/193784/Informe\%20nacional\%20de\%20resultados\%202014\%20 II\%202017\%20II\%20saber\%2011.pdf.

Instituto Nacional de Estudos e Pesquisa Educacionais Anisio Texeira [Inep]. Resultado do Enem 2019 é divulgado pelo Inep; saiba como consultar a nota, 2019. Recuperado de https:/g1.globo.com/educacao/enem/2019/noticia/2020/01/17/notas-do-enem2019-sao-divulgadas-pelo-inep.ghtml.

Johnson, Ralph H. Manifest rationality. A pragmatic theory of argument. Mahwah, NY: Lawrence Erlbaum, 2000.

Kuhn, Deanna y Pearsall, Susan. "Developmental Origins of Scientific Thinking”. Journal of Cognition and Development, (2000). 113-129. https://doi.org/10.1207/ S15327647JCD0101N_11. 
Kuhn, Deanna y Udell, Wadiya. “The Development of Argument Skills”. Child Development, 74(5), (2003): 1245-1260. https://doi.org/10.1111/1467-8624.00605.

Kuhn, Deanna. "A role for reasoning in a dialogic approach to critical thinking". Topoi, 37(1), (2018): 121-128. doi: https://doi.org/10.1007/s11245-016-9373-4.

Kuhn, Deanna y Franklin, Sam. The Second Decade: What Develops (and How). En D. Kuhn, R. S. Siegler, W. Damon, y R. M. Lerner (Eds.), Handbook of child psychology: Cognition, perception, and language (pp. 953-993). John Wiley y Sons Inc, 2006.

Kuhn, Deanna, Hemberger, L., y Khait, V. Dialogic argumentation as a bridge to argumentative thinking and writing. Infancia y Aprendizaje, 39(1), (2016): 25-48. https:// doi.org/10.1080/02103702.2015.1111608.

Kuhn, Deanna. The skills of argument. Cambridge: Cambridge University Press, 1991.

Larraín, Antonia; Freire, Paulina; Moretti, Renato; Requena, Magdalena y Sabat, Belén. “La universidad de Chile promueve las habilidades de argumentación escrita? Un estudio exploratorio comparativo de estudiantes de educación universitaria y educación técnica". Calidad en la educación, (43), (2015): 201-228. doi: http://dx.doi. org/10.31619/caledu.n43.49.

Larson, Aaron; Britt, M. Anne y Kurby, Christopher A. "Improving Students' Evaluation of Informal Arguments". The Journal of Experimental Education, 77:4, (2009): 339366, doi: 10.3200/JEXE.77.4.339-366.

Latorre, Diana. Avaliação de argumentos no ensino superior: transferência de habilidades da sala de aula a situações extracurriculares. Diss. Universidade Federal de Pernambuco, Recife, 2018.

Leitão, Selma. "The potential oh argument in knowledge building”. Human Development, 6, (2000): 332-360. doi: 10.1159/000022695.

Leitão, Selma. "Argumentação e desenvolvimento do pensamento reflexivo". Psicología: Reflexão e Crítica, 20(3), (2007): 454-462. https://doi.org/10.1590/S010279722007000300013.

Leitão, Selma. La dimensión epistémica de la argumentación. En E. Kronmüller y C. Cornejo, La pregunta por la mente: aproximaciones desde Latinoamérica (pp. 87-119). Santiago de Chile: JCSaez Editor, 2008.

Leitão, Selma. "O trabalho com argumentação em ambientes de ensino-aprendizagem". Uni-pluri/versidad, 12(3), (2012): 23-37. https://revistas.udea.edu.co/index.php/ unip/issue/view/1349.

Lipman, Matthew. Thinking in education. Cambridge, MA: Cambridge University, 1993. 
Lozano, Marcela. Estrategia Nacional de Apropiación Social de la Ciencia, la Tecnología y la Innovación -Colciencias. Foro-Taller Apropiación Social de la Ciencia y la Tecnología, 2010.

Marafioti, Roberto. Argumentando acerca de la argumentación. En: R. D. Aires (Ed.), Anales de la Educación Común (pp. 1-10). Buenos Aires: Tercera Época, 2007.

Meneses Alba, Julie X.; Osorio Castañeda, Katherine y Rubio Quintero, Ana M. "La comprensión de textos argumentativos como estrategia para el aprendizaje significativo y el desarrollo del pensamiento crítico". Actualidades Pedagógicas, (72), (2018): 29-47. doi: https://doi.org/10.19052/ap.4336.

Miller, Brian W.; Anderson, Richard; Morris, Joshua; Lin, Tzu-Jung; Jadallah, May y Sun, Jingjing. "The effects of reading to prepare for argumentative discussion on cognitive engagement and conceptual growth". Learning and Instruction, 33, (2014): 67-80. doi: 10.1016/j.learninstruc.2014.04.003.

Nussbaum, Michael y Sinatra, Gale. "Argument and conceptual engagement". Contemporary Educational Psychology, 28(3), (2003): 384-395. doi: 10.1016/S0361476X(02)00038-3.

Programme for International Student Assessment [PISA]. 2018. Recuperado de: https:// www.oecd.org/pisa/publications/pisa-2018-results.htm.

Ramírez, Nancy. Desenvolvimento do pensamento reflexivo: avaliação da qualidade da argumentação em situação de debate crítico. Diss. Universidade Federal de Pernambuco, Recife, 2012.

Ramírez, Nancy; Souza, Dayse y Leitão, Selma. "Desarrollo de habilidades argumentativas en la enseñanza-aprendizaje de contenidos curriculares". Cogency, 5(1), (2013): 107-133. http://www.cogency.udp.cl/index.php/cogency/issue/view/27.

Rapanta, Chrysi; Garcia-Mila, Merce y Gilabert, Sandra. "What Is Meant by Argumentative Competence? An Integrative Review of Methods of Analysis and Assessment in Education". Review of Educational Research, XX, X, (2013): 1-38. doi: 10.3102/0034654313487606.

Rennau, Nancy. “The Debate Debate". The Clearing House, 78(2), (2004): 52-55. doi: 10.3200/TCHS.78.2.52-56.

Rogoff, Barbara. The cultural nature of human development. Oxford: Oxford University Press, 2003.

Saíz, Carlos y Rivas, Silvia. "Evaluación en Pensamiento Crítico: Una Propuesta para Diferenciar Formas de Pensar”. Ergo, Nueva Época, 22/23, (2008): 25-66. http://cdigital. uv.mx/handle/123456789/38320. 
Saíz, Carlos. “Enseñar o aprender a pensar”. Escritos de Psicología, 6, (2002): 53-72.

Super, Charles M. y Harkness, Sara. "Culture structures the environment for development". Human Development, 45, (2002): 270-274. http://dx.doi.org/10.1159/000064988.

Torres, Doris; Fonseca, Widy y Pineda, Blanca. "Las vivencias como estrategia de fortalecimiento del pensamiento crítico en educación rural". Praxis y Saber, 8(17), (2017): 201-224. https://doi.org/10.19053/22160159.v8.n17.2018.7207.

Valsiner, Jaan. "Process Structure of Semiotic Mediation in Human Development". Human Development, 44, (2001): 84-97. doi: 10.1159/000057048.

Valsiner, Jaan. Culture in Minds and Societies: Foundations of Cultural Psychology. London, 2006.

Van Eemeren, Frans H. y Grootendorst, Rob. Rules for a critical discussion. En F. H. Van Eemeren y R. Grootendorst. A Systematic Theory of Argumentation: The Pragmadialectical Approach, Cambridge, Cambridge University Press, 2004.

Van Eemeren, Frans H.; Grootendorst, Rob y Snoeck, A. F. Unexpressed Standpoints and unexpressed premises. En: F. H. Van Eemeren; R. Grootendorst y A. F. Snoeck. Argumentation: Analysis, Evaluation, Presentation. p.p. 49-57. Mahwah, Nj: Taylor y Francis. 2008.

Van Eemeren, Frans H. "Consideren la brecha”: Conciliando la búsqueda del éxito con la persistencia de la razonabilidad. En Santibañez, C. y Riffo, B. Estudios en argumentación y Retórica. Teorías contemporâneas y aplicaciones. Chile: Editorial Universidad de Concepción, 2007.

Voss, James F. y Van Dyke, Julie A. "Argumentation in Psychology: Background Comments". Discourse Processes, 2-3(32), (2001): 89-111. https://doi.org/10.1080/0163 853X.2001.9651593.

Walton, Douglas. "Commitment, Types of Dialogue, and Fallacies". Informal Logic, 14(2), (1992): 93-103. doi: https://doi.org/10.22329/il.v14i2.2532.

Walton, Douglas. "A dialogue model of belief". Argument \& Computation, 1(1), (2010): 23-46. https://doi.org/10.1080/19462160903494576.

Wang, Qi y Brockmeier, Jens. "Autobiographical remembering as cultural practice: Understanding the interplay between memory, self and culture". Culture \& Psychology, 8, (2002): 45-64. http://dx.doi.org/10.1177/1354067X02008001618.

Wertsch, James V. From social interaction to higher psychological processes: A clarification and application of Vygotsky's theory. Human Development, 51, (2008/1979): 66-79. Reprint from Human Development, 22, 1-22. doi: 10.1159/000272425. 
68 - Cogency, Journal of reasoning and argumentation

Wolfe, Christopher R. "Individual Differences in the "Myside Bias" in Reasoning and Written". Argumentation Written Communication 29(4), (2012): 477-501. doi: $10.1177 / 0741088312457909$.

Yin, Robert. Case study research: design and methods (3 ed., Vol. 5). Londom: Sage Publications, Lta, 2002. 